\title{
EL USO SOSTENIBLE DE LA TIERRA EN LOS BOSQUES DE LA LLANURA ALUVIAL INUNDABLE PERUANA: OPCIONES, PLANEAMIENTO E IMPLEMENTACION ${ }^{1}$
}

\section{Gustav Nebel ${ }^{2}$}

\section{RESUMEN}

La llanura aluvial inundable comprende más del 12\% de la selva baja de la Amazonía Peruana y es económicamente importante en la agricultura, pesca, caza, actividades forestales y aprovechamiento de otros productos forestales. La presión sobre los recursos de la llanura aluvial inundable está en aumento, por tanto es necesario planear e implementar el uso apropiado de la tierra. Este artículo describe algunas características especiales de las llanuras aluviales inundables e interpreta sus implicancias en el uso de la tierra. Existe una estrecha interacción entre los ecosistemas terrestres y acuáticos de las llanuras aluviales inundables, lo que refuerza la necesidad de un manejo a fin de poner especial atención a este atributo. Por naturaleza, las llanuras aluviales inundables están adaptadas a grandes perturbaciones, lo cual indica que se pueden llevar a cabo intervenciones de manejo. Sin embargo, se nota que solamente una limitada proporción de la tierra, dentro de cada hábitat, es perturbada a la vez, lo cual sugiere que se debe evitar la conversión -a gran escala- de ciertos hábitats de bosque. La mayor fertilidad de los suelos de la llanura aluvial inundable y los nuevos ingresos periódicos de material fértil, debido a la sedimentación, sugiere que el riesgo de deterioro del suelo es limitado. El potencial de producción de las llanuras aluviales inundables es relativamente alto, aunque varía de acuerdo a los patrones de inundación y drenaje, reforzando la necesidad de proporcionar información sobre la variación geográfica en estos factores ambientales. La cultura y organización de los

1 Traducción del original en inglés "Sustainable land-use in peruvian flood plain forests: options, planning and implementation".

2 Royal Veterinary and Agricultural University. Department of Economics and Natural Resources. Unit of Forestry, Rolighedsvej 23, 1958 Frederiksberg C.,Denmark. Phone: 45 352822 32, Fax: 45352826 71. E-mail: gne@kvl.dk 
habitantes de la llanura aluvial inundable ha sido dinámica, con el dominio de un sistema-patrón en muchas actividades económicas. Pocas normas legales y administrativas controlan el acceso y el uso de los recursos naturales de la llanura aluvial inundable y no se puede otorgar derechos permanentes de tenencia de la tierra en dichas áreas. Estas condiciones agudizan la necesidad de promover organizaciones e instituciones adaptadas a estas condiciones y de desarrollar un sistema de tenencia de la tierra otorgando incentivos por las actividades de manejo, con una perspectiva de largo plazo. Se descubren diferentes opciones del uso de la tierra de la llanura aluvial inundable, adaptadas a la producción de productos forestales maderables y no maderables de los bosques naturales y se discute la domesticación de recursos forestales. Se encontró que la producción de madera, sobre la base de un rendimiento sostenido en estos bosques, tiene un valor presente neto de hasta 250 US $\$ / h a$, sin la incorporación de tratamientos de mejora del crecimiento a partir de posibles raleos y tratamientos de liberación. En promedio, el valor real del aprovechamiento de productos forestales no maderables es bajo, pero ciertos hábitats generan beneficios económicos muy altos; asimismo, el aprovechamiento de esos productos constituye la fuente principal de la economía de muchos pobladores de la llanura aluvial inundable. La domesticación de las especies arbóreas y arbustivas tiene un alto potencial en las llanuras aluviales inundables y la agroforestería ya es una actividad difundida. Los efectos de la inundación, el mal drenaje y el riesgo de erosión por la corriente del río son impedimentos para la implementación de sistemas exitosos de uso intensivo de la tierra y se puede correr el riesgo de tener distorsiones económicas si la tierra, que es apropiada para una producción agrícola de subsistencia, es convertida gradualmente, por otros agricultores, en tierras de producción comercial a gran escala.

Palabras clave: Zonas inundables, ecología, socio-economía, forestería, agroforestería, productos forestales, productos forestales no maderables, valor presente neto.

\section{ABSTRACT}

Flood plains comprise more than $12 \%$ of the lowland Peruvian Amazon, and they are economically important in agriculture, fishing, hunting, forestry and extraction of other forest products. The pressure on the flood plain resources is increasing, and planning and implementation of adapted land-uses is needed. This paper outlines some special features of the flood plains and interpret their implications for landuse. A close interaction exists between terrestrial and aquatic ecosystems of the flood 
plains, stressing the need for management to pay special attention to this attribute. By nature the flood plains are adapted to large perturbations, indicating that rough management interventions can be carried out. However, it is noticed that only a limited proportion of the land within each habitat is disturbed by nature at a time, suggesting that, for example, large-scale conversion of certain forest habitats must be avoided. The generally high fertility of the flood plain soils and periodical new inputs of fertile material from sedimentation hint that the risk of soil depletion is limited. The production potential of the flood plain is relatively high, although it varies according to patterns of flooding and drainage, stressing the need to provide information on the geographic variation in these environmental factors. The culture and organization of flood plain inhabitants have been dynamic, and a pattern system prevails in many economic activities. Only few legislative and administrative regulations control the access to and use of the flood plain natural resources, and no permanent tenure rights can be issued for hand in the flood plain areas. These conditions emphasize the need to promote adapted organizations and institutions, and to develop an land tenure system fostering incentives for management working on a long-term perspective. Different flood plain land-use options encompassing production of timber and nontimber forest products from natural forests, and the domestication of flood plain forest resources are described and discussed. It is found that timber production on a sustained yield basis in the natural flood plain forests has net present values up to $250 \mathrm{US} \$ /$ ha in systems where growth enhancing effects from possible thinning and liberation treatments are not accounted for. On the average, the value of actual extraction of non timber forest products is low, but certain habitats generate very high economic benefits, and the extraction of these products constitute a major part of the economy of many flood plain households. Domestication of forest tree and bush species has high potential in the flood plains, and agroforestry is already widespread. Effects of flooding, bad drainage and risk of erosion by the river current are impediments to successful intensive land-use systems, and socio-economic distortions can be risked if land suited for agricultural subsistence production is gradually taken over by agriculturalist focussing on large-scale commercial production.

Key words: Flood plain, ecology, socio-economics, forestry, agroforestry.

\section{INTRODUCCION}

La selva baja de la Amazonía Peruana abarca alrededor de $680000 \mathrm{~km}^{2}$ (Dourojeanni, 1990). En esta área, los ríos constituyen la más importante infraestructura así como los llanos inundables y los ríos ricos en sedimentos constituyen más del $12 \%$ de la 
superficie (Salo et al., 1986). Una gran parte de la población rural está establecida cerca o en la misma llanura aluvial inundable, lugar en el cual desarrollan sus actividades económicas como la agricultura, la pesca, la caza, la forestería y la extracción de otros productos forestales (Kvist et al., 1995, 1999a, 1999b; Kvist y Nebel, 1999). La presión sobre los recursos naturales de la llanura aluvial inundable está aumentando debido a las intervenciones a gran escala y a la sobre-explotación de ciertas poblaciones de plantas y animales (Soini et al., 1996; Kvist y Nebel, 1999; Kvist et al., 1999a). Simultáneamente, los intentos de planeamiento del uso de la tierra así como de manejo y conservación de los recursos naturales, han sido ineficientes o no existen. Son múltiples las causas esenciales de esta situación e incluyen factores tales como una población en aumento, que demanda un mejor bienestar económico; la alteración de las normas tradicionales del uso del recurso natural; la falta de un compromiso político y de capacidad para planificar y reglamentar la extracción.

El presente artículo delinea algunas características de la llanura aluvial inundable; a la vez, interpreta las implicancias de su uso con el propósito de apoyar el desarrollo y la implementación de sistemas apropiados de manejo de estos ecosistemas. Se describen y discuten las posibilidades de diferentes usos de la tierra y se dan algunas recomendaciones para el planeamiento y la implementación de un uso sostenible. Se pone énfasis en cuestiones ecológicas y en las condiciones que diferencian los bosques de la llanura aluvial inundable de otros tipos de bosques húmedos tropicales.

\section{PROPIEDADES DE LOS LLANOS INUNDABLES Y SUS IMPLICANCIAS EN EL USO DE LA TIERRA}

Las inundaciones tienen un profundo impacto ecológico en los ecosistemas de la llanura aluvial inundable amazónica. Los elementos de los sistemas terrestres y acuáticos interactúan y a menudo ambos sistemas se benefician con la asociación, especialmente cuando el ritmo de la inundación es regular (Junk et al., 1989; Junk, 1997). El manejo debería considerar explícitamente este atributo, al integrar las consideraciones de posibles efectos en el otro sistema. Por ejemplo, las actividades agrícolas y forestales deben prestar atención a sus posibles efectos en la alimentación y la reproducción de las poblaciones de peces.

Los procesos fluviales causan considerables perturbaciones ambientales en la llanura aluvial inundable. La continua erosión de las riberas de los ríos y los cambios repentinos en los cursos de los ríos devastan la vegetación existente. Mientras tanto, la sedimentación trae consigo la formación de nuevas tierras, las cuales dan lugar al 
establecimiento y la sucesión de la vegetación. Estos procesos operan usualmente en una pequeña extensión de terreno, mientras que los cambios menos frecuentes, debido al desplazamiento del curso del río, causan cambios en el drenaje y la sedimentación sobre áreas más grandes (Salo y Räsänen, 1989). El paisaje resultante es un mosaico complejo, en el tiempo y en el espacio, de hábitats entremezclados que se componen de diferentes ambientes y etapas de sucesión (por ej. Foster et al., 1986; Foster, 1990; Gentry y Terborgh, 1990; Worbes et al., 1992; Kalliola y Puhakka, 1993; Worbes, 1997; Kvist y Nebel, 1999). A menudo, los procesos de crecimiento en los bosques del llano inundable devienen en un considerable retorno del bosque (Nebel et al., 1999a). Por lo tanto, se cree que los ecosistemas de la llanura aluvial inundable están adaptados a grandes perturbaciones, lo que indica que se pueden llevar a cabo leves intervenciones de manejo, dentro del rango de los procesos que naturalmente ocurren. Sin embargo, debe notarse que dentro de cada tipo de hábitat, solamente una limitada proporción de la tierra es perturbada por la naturaleza en un tiempo dado, lo cual sugiere que los ecosistemas no están adaptados a disturbios extensos simultáneos. Esto significa que las interferencias severas deben estar restringidas a una limitada proporción de cada hábitat. Con el fin de manejar los frecuentes pequeños espacios de los diferentes hábitats de la llanura aluvial inundable, debe aplicarse una propuesta flexible y de pequeña escala que incida en el manejo.

Las llanuras aluviales inundables amazónicas, así como los principales ríos de agua turbia, son de formación relativamente reciente (Räsänen et al., 1987, 1992; Irion, 1989; Räsänen, 1993; Irion et al., 1997) y los suelos son típicamente fértiles con un alto contenido de nutrientes valiosos y con nuevas contribuciones periódicas (por ej. Fageria et al., 1991; Furch, 1997). Esto sugiere que la biomasa puede ser removida sin tener mucho en cuenta la disminución de la fertilidad del suelo (Furch, 1997). La producción potencial es relativamente alta, aunque varía en varios grados, dependiendo de las presiones relacionadas a la inundación y al drenaje. Nebel et al. (1999b) observaron, en los bosques de la llanura aluvial inundable peruana, considerables tasas de crecimiento de árboles y una relativamente alta producción primaria neta de la biomasa forestal leñosa. Esto sugiere que existe un gran potencial para la producción de madera; sin embargo, la fertilidad del suelo y los patrones de drenaje han cambiado considerablemente, evidenciándose la necesidad de mayor información sobre su variación espacial. La latencia cambial de las especies leñosas de la llanura aluvial inundable, como resultado de las adaptaciones morfológicas y fisiológicas, es quizá la principal respuesta de sobrevivencia a la inundación (Gill, 1970; Crawford, 1982; Hook, 1984; Junk, 1989; Schlüter et al., 1993; Armstrong et al., 1994; Worbes, 1997). En términos de predicción de la amplitud y la duración, el patrón de inundación es decisivo en la composición florística y en el potencial de producción de biomasa 
de lugares específicos. Algunos procesos ecológicos importantes, como la dispersión, la depredación y la germinación de semillas, así como los herbívoros que atacan a las plántulas, pueden también ser influenciados por el patrón de inundación. La ocurrencia de hechos extremos, como el de años consecutivos de alta y larga inundación, son raramente cruciales para el carácter del ambiente y, consecuentemente, para la composición y estructura de la vegetación (Junk, 1989; Irion et al., 1997; Junk y Piedade, 1997). El manejo en las llanuras aluviales inundables debe considerar esta estratificación ambiental, lo que implica que ciertos espacios son más apropiados para una especie y tipos de producción particulares. Esto otorga por condición, la necesidad de sistemas de manejo capaces de controlar situaciones de hábitats de pequeños espacios. Una importante herramienta para el manejo de la llanura aluvial inundable, sería también el contar con una clasificación geográfica concordante con las características de los patrones de inundación.

Las plantas y animales de las llanuras aluviales inundables de la Amazonía son generalmente diversos (Goulding, 1983; Kubitzki, 1989). Aún más, muchas de las especies parecen estar adaptadas a la dispersión y migración sobre grandes distancias, a menudo a través de los canales abiertos del agua (Gottsberger, 1978; Goulding, 1980, 1985; Kubitzki, 1989; Goulding et al., 1996; Junk et al., 1997; Petermann, 1997; Worbes, 1997). Esto sugiere que las especies son genéticamente algo uniformes, aunque las restricciones, en la dispersión aguas arriba, puede favorecer la formación de sub-poblaciones. A partir de esto, se puede construir la hipótesis de la existencia de un limitado riesgo de pérdida de información genética, debido a la sobre-explotación, de las poblaciones de la llanura aluvial inundable, pero esta hipótesis tiene que ser verificada experimentalmente.

Los bosques de la llanura aluvial inundable proporcionan recursos para el sostén de una gran parte de la población rural de la Amazonía Peruana. Las actividades socioeconómicas varían, desde la extracción de productos directamente vendibles, hasta las operaciones de subsistencia como la pesca, la caza o la recolección de productos forestales. El amplio rango de usos del recurso natural está ligado a la gran diversificación de hábitats, ya que los diferentes tipos de bosque proveen posibilidades para distintas actividades y la extracción se ve estimulada por una frecuente alta densidad de los recursos. En el complejo escenario de la llanura aluvial inundable, la composición de los hábitats, fácilmente alcanzable por los extractores, determina, en gran medida, el portafolio de las actividades socio-económicas (Kvist y Nebel, 1999; Kvist et al., 1999b). La cultura y la organización de los habitantes del llano inundable peruano son dinámicas. Ha ocurrido un gran cambio desde el dominio de las bien organizadas comunidades indígenas hasta el arribo de los europeos (Meggers, 1971; 
Denevan, 1976; Moran, 1989; Roosevelt, 1989) y las actuales comunidades dominadas por mestizos, fuertemente influenciadas por factores tales como la migración de gente joven capacitada y las fuertes campañas religiosas que crearon nuevas normas (por ej. Hiraoka, 1985a; Kvist y Nebel, 1999). El feudalismo ha sido extensivo y ha dominado la organización rural durante el periodo de la extracción del caucho, a comienzos del siglo XX (por ej. Collier, 1981; Pennano, 1988). El derecho de los más fuertes todavía prevalece y la extracción comercial de los recursos de la llanura aluvial inundable continúa siendo organizada y realizada por la gente de la ciudad, creando solamente limitados retornos económicos a las comunidades rurales. En un contexto de manejo de las condiciones arriba mencionadas, es importante adquirir conocimientos sobre la capacidad de carga de los diferentes hábitats de la llanura aluvial inundable y, consecuentemente, de su valor socio-económico potencial. El desarrollo y la elaboración de herramientas que apoyen el planeamiento del eficiente uso de la tierra es de principal importancia en esta relación (por ej. la clasificación de hábitats, los mapas, etc.). Además, es necesario la promoción de estructuras de organización apropiadas para el manejo de los pocos recursos los cuales, casi siempre, están al alcance de mucha gente.

El acceso y uso de los recursos naturales de la llanura aluvial inundable peruana es controlado por pocas normas legales y administrativas y aquéllas que se han dado han sido muy raramente cumplidas. La extracción de la madera comercial está formalmente permitida a través del sistema de concesiones, lo cual ha favorecido a los pequeños dueños de concesiones de corto plazo de hasta 1000 ha (FAO, 1993). Un gran número de extractores opera en tales concesiones, usando típicamente técnicas primitivas de apeo y transporte de trozas (Kvist y Nebel, 1999). A menudo, estos extractores operan fuera de las áreas especificadas y cortan árboles por debajo de los diámetros mínimos de corta legalmente especificados. Solamente algunos pocos agricultores poseen licencia de tenencia provisional de las áreas de la llanura aluvial inundable que cultivan; además, la actual distribución de los derechos de los usuarios se basa, principalmente, en las tradiciones y normas locales. La legislación sobre las tierras en Perú está bajo revisión y se prevé la titulación de la mayoría de la selva baja amazónica peruana mediante la subasta pública. Sin embargo, parece que se excluye a la zona de la llanura aluvial inundable debido a que este tipo de terreno está sujeto a procesos continuos de cambios de forma. Esta situación es lamentable puesto que el éxito del manejo y la conservación depende de un organismo legislativo firme y transparente relacionado con los derechos de tenencia. 


\section{OPCIONES DEL USO DE LA TIERRA EN LOS BOSQUES DE LA LLANURA ALUVIAL INUNDABLE PERUANA}

\subsection{Producción de madera en los bosques naturales}

Una gran proporción de la madera que actualmente se extrae en la Amazonía Peruana proviene de los bosques inundables (Kvist y Nebel, 1999). Debido, generalmente, a las condiciones ecológicas favorables parece que se pueden aplicar varios sistemas de manejo dirigidos a producir madera. Sin embargo, deben ser excluidos algunos hábitats de la llanura aluvial inundable debido, principalmente, a la inundación prolongada o impredecible y al pobre drenaje. Según Kvist y Nebel (1999), tales hábitats incluyen las tahuampas de cuencas inundables y los bosques de bajial. En estos tipos de bosque, la densidad de las especies deseables es baja y su producción potencial y capacidad regenerativa parecen limitadas (Freitas, 1996; Kvist y Nebel, 1999). Sin embargo, la comercialización en el mercado de nuevas especies y el mejor conocimiento de las técnicas que estimulan el establecimiento y el crecimiento de especies deseables, podrían cambiar la situación.

Puesto que las profundas y extendidas perturbaciones de las llanuras aluviales inundables no suceden naturalmente y pueden tener consecuencias adversas desconocidas, se sugiere que los ecosistemas forestales no sean excesivamente manipulados abarcando grandes áreas. Por lo tanto, los sistemas silviculturales policíclicos, caracterizados por una extracción periódica y selectiva de un número limitado de árboles maduros del bosque natural, parecen ser la mejor opción para una producción comercial a gran escala en los bosques de la llanura aluvial inundable peruana. Bajo tales sistemas, la composición florística y la estructura de los bosques son ligeramente manipuladas, lo que mantiene la estabilidad del ecosistema y el funcionamiento en el tiempo y el espacio. La producción de madera en estos sistemas también considera la provisión de otros productos y servicios forestales, que ayudan a balancear económicamente el rendimiento más alto en madera conseguido en otros sistemas silviculturales. La baja demanda de capital y los retornos económicos, relativamente rápidos, que caracterizan a los sistemas policíclicos, son otras de sus ventajas ya que el capital es escaso en la economía peruana en vías de desarrollo.

Los sistemas policíclicos apropiados para los bosques de la llanura aluvial inundable peruana aún necesitan ser desarrollados, pero ya se pueden identificar algunas características. Es posible transportar la madera desde los ríos cercanos al lugar de extrac- 
ción, ya sea en chatas o en balsas flotantes, con trozas de especies de baja densidad. En épocas de inundación, las trozas más ligeras pueden llevarse a flote desde los lugares de extracción hasta los ríos y luego, desde allí, ser posteriormente transportadas; también se pueden usar ventajosamente otras técnicas de baja tecnología como el arrastre con búfalos, considerando que el terreno de la llanura aluvial inundable es plano y las distancias a los cursos de agua son normalmente cortas debido a la gran cantidad de quebradas. Llevar por flotación las trozas desde el lugar de corta es la manera más simple de extraerlas, pero eso implica el riesgo de perder las trozas ya cortadas en caso de que ocurriera una baja o breve inundación. El talado está restringido a un periodo relativamente corto, previo a la inundación, con el fin de prevenir el deterioro de la madera, limitando así la capacidad de los equipos de extracción. Adicionalmente, si se requiriera colectar plántulas de las especies extraídas sería problemático talar árboles antes de la inundación, puesto que muchas especies fructifican durante y poco después de la inundación. En estos casos el talado debe ser llevado a cabo lo más pronto posible después de la inundación a fin de permitir el máximo crecimiento de las plántulas, lo que incrementa su resistencia a la siguiente inundación. Comparado con los sistemas de transporte mecanizado convencionales y el transporte con tractores de trozas, estas técnicas reducen significativamente los costos de extracción (Barros y Uhl, 1995) así como los daños por arrastre del rodal remanente.

Hasta ahora existen pocos resultados que pueden ser usados para evaluar el nivel del rendimiento sostenido de madera, a partir de los sistemas silviculturales policíclicos, en los bosques de la llanura aluvial inundable amazónica. Sin embargo, las recomendaciones fueron provistas por los datos obtenidos en nueve parcelas de muestreo permanentes de una hectárea, en los bosques de la llanura aluvial inundable de Braga-Supay y Lobillo de la Amazonía Peruana. El stock en pie de las especies de madera comercial, mayores o igual a $10 \mathrm{~cm}$ DAP, fue alto $\left(59-286 \mathrm{~m}^{3} / \mathrm{ha}\right.$ ) y durante el periodo de 1993 a 1997 hubo un considerable incremento en volumen de estas especies en las parcelas de muestreo tratadas y no tratadas (2-9 $\mathrm{m}^{3} / \mathrm{ha} / \mathrm{año}$; Nebel et al., 1999a). El volumen total de todas las especies mayores o igual a $10 \mathrm{~cm}$ DAP en los bosques fue de $618-773 \mathrm{~m}^{3} /$ ha y la producción primaria neta de la biomasa maderable fue alta (1 $709 \mathrm{~g} / \mathrm{m}^{2} / \mathrm{año}$; Nebel et al., 1999b). La alta producción forestal abarca una considerable variación en el crecimiento de las especies individuales, demostrándose por los altos niveles de incremento en madera para un especie forestal emergente, utilizada para madera contrachapada (Nebel et al., 1999c), y por una modesta tasa de crecimiento para una especie de dosel medio que proporciona una madera de alta densidad (Nebel, 1999). 
Con el fin de evaluar posteriormente el rendimiento sostenido de madera de los bosques de la llanura aluvial inundable de Braga-Supay y Lobillo, se desarrollaron cinco escenarios que muestran diferentes intensidades de aprovechamiento. Se estableció, para cada especie maderable comercial, un diámetro mínimo de aprovechamiento (MHD) imaginario correspondiente al 60, 70, 80, 90 y 100\% del diámetro máximo a la altura del pecho (DAP) alcanzado por los individuos de las especies. A fin de prevenir la simulación del exceso de extracción de las especies que se desarrollan a tamaños relativamente pequeños se aplicaron MHD específicos de cada especie. Se evaluaron algunos parámetros importantes para cada uno de los cinco escenarios de los tres tipos de bosque investigados (Figura 1). En cada escenario se calcularon el número y área basal promedio de los árboles comerciales que excedieron los MHD (Figura 1a, 1b), lo que resultó en un aprovechamiento simulado de hasta 22 árboles/ ha correspondiendo al $22 \%$ del área basal total. De los aprovechamientos simulados, se obtuvieron considerables volúmenes de madera extraída y en el apeo más intenso, en el bosque de restinga alta, se cortó aproximadamente $150 \mathrm{~m}^{3} /$ ha (Figura 1c). El incremento de las especies maderables comerciales, en las parcelas permanentes de muestreo de los tres bosques de la llanura aluvial inundable, fue relativamente alto (Figura 1d), dando lugar a ciclos de corta de hasta 55 años en los casos en que el rendimiento en madera debió ser sostenido (Figura 1e). Las utilidades netas por la extracción maderera fueron relativamente altas (Figura 1f), aunque es posible tener costos más bajos si se transportan las trozas en balsas en lugar de barcazas, tal como se asume en los cálculos. En suma, el valor capitalizado del aprovechamiento permanente, con rendimiento sostenido de madera, aplicando corta de lianas trepadoras, mapeo de árboles maderables y tres intervenciones de raleo durante cada ciclo de corta, fue bajo (Figura 1g). Sin embargo, para las tasas de interés aplicadas, la relación beneficio/costo de la extracción maderera fue positiva en los tres tipos de bosque (Figura 1h).

\subsection{Productos no maderables provenientes de bosques naturales}

En las llanuras aluviales inundables peruanas existen muchos productos forestales no maderables (PFNM) que provienen de una variedad de plantas y animales originarios o dependientes de los bosques naturales de la llanura aluvial inundable. Estos contribuyen significativamente a la sobrevivencia de casi todos los habitantes de la llanura aluvial inundable (Kvist et al., 1995, 1999a, 1999b). En un estudio de los comuneros de siete comunidades de la llanura aluvial inundable, Kvist et al. (1999b) encontraron que más del $60 \%$ del valor total estimado de las economías de los campesinos entrevistados se debía a la extracción, principalmente de PFNM, y casi la 
mitad de su ingreso monetario era generado por esta fuente. Los productos provenientes de animales eran responsables de más del $60 \%$ del valor de los productos extraídos, tanto para la subsistencia como para su comercialización, y en ambos casos la pesca era la más importante. Las actividades de subsistencia recaían generalmente en un rango más amplio de especies que en las actividades comercialmente dirigidas, en donde unas cuantas especies importantes constituían la columna vertebral de la extracción, aunque esto variaba mucho de acuerdo a la abundancia local de los recursos (Kvist et al., 1999a, 1999b). En algunos casos, esto ha devenido en una disminución local o regional de las especies comercialmente explotadas (Kvist y Nebel, 1999). Los patrones observados concuerdan con Wilkie y Godoy (1996), quienes sugirieron que la extracción de recursos exportables, probablemente, causan una disminución en el número de especies extraídas y en la sobre-extracción. De la misma manera, la extracción de muchos PFNM de las llanuras aluviales inundables peruanas parecen seguir un ciclo económico para la extracción de productos forestales (propuesto por Homma, 1996), el cual consiste en el desarrollo cronológico de las fases de expansión, estabilización, decaimiento y cultivo.

Kvist et al. (1999b) encontraron áreas promedio de propiedades de 100-158 ha, donde se desarrollan actividades de extracción de PFNM que proporcionan ingresos de 8-15 US\$/ha/año. Consecuentemente, el VPN por hectárea de la extracción de PFNM de las llanuras aluviales inundables es más bajo, debido a que los costos de extracción necesitan ser restados de los valores mencionados. Sin embargo, estas cifras eran promedios de la extracción en áreas circulares con una extensión que iba desde el caserío de los extractores hasta el límite donde ellos conducían sus actividades. Por lo tanto, las cifras promedio cubrían las enormes variaciones en la importancia de los hábitats específicos, así como del área usada y del valor fijado por los comuneros y las comunidades. Por ejemplo, ciertos lagos o rodales forestales dominados por palmeras generaban gran parte de los valores. Más aún, los comuneros, así como las comunidades, tendían a especializarse en la extracción de productos de los cuales tenían un conocimiento particular o de aquéllos con extraordinaria abundancia en el área. Al crear los incentivos económicos por la extracción de muchas categorías de PFNM, es de gran importancia la localización geográfica de los hábitats utilizados en la extracción de PFNM en relación a los comuneros; también lo es una infraestructura que proporcione un eficiente y efectivo costo de transporte de productos a los mercados regionales. Peters et al. (1989) y Muñiz-Miret et al. (1996) registraron grandes ingresos anuales por la extracción en los hábitats de la llanura aluvial inundable dominada por palmeras, originando VPN estimados que van desde 5702 a 11265 US $\$$ ha al $5 \%$ de tasa de interés. En ambos casos, la proximidad a los mercados locales fue esencial para obtener altos VPN. Estas condiciones acentúan la necesidad de dirigir el manejo a hábitats específicos y a especies que contribuyan, en gran 
parte, al valor de la extracción. Igualmente, se puntualiza que se debe tener cuidado de no exagerar los beneficios de la extracción de PFNM basándose en los estudios concentrados en las zonas más valiosas de los bosques de la llanura aluvial inundable. Mientras tanto, la extracción de los PFNM permanece como un ventaja económicamente importante en las economías de los campesinos de la llanura aluvial inundable rural.

Desde el punto de vista biológico y técnico, algunos aspectos centrales a ser atendidos involucran el conocimiento de cada PFNM en lo que se refiere a la identificación de la especie, rendimiento potencial e incidencia geográfica. La apropiada identificación de las especies es un pre-requisito para planear la mayoría de los esfuerzos de manejo. La tarea puede parecer muy simple, pero en los complejos bosques tropicales aún persisten muchos problemas taxonómicos sin resolver. En algunos casos, algunos PFNM importantes provienen de especies pobremente conocidas científicamente, pero el problema más común de identificación es aquél en que un solo nombre vernacular comprende varias especies que proporcionan el mismo producto. En los bosques del llano inundable peruano, todavía existe confusión en los nombres de las especies de algunos PFNM, pero -generalmente- sucede en especies usadas con fines de subsistencia (Kvist et al., 1999a). El rendimiento potencial de los PFNM puede ser evaluado de diferentes maneras e interactúa estrechamente con la estructura de la población de la especie en cuestión la cual -a su vez- está influenciada por los impactos de la extracción y el monitoreo de la regeneración y los rodales. Los modelos de crecimiento pueden proporcionar buenas estimaciones del rendimiento potencial de las poblaciones de especies de PFNM, pero este método requiere de datos que raramente están disponibles. Alternativamente, el desarrollo en la categoría de las poblaciones de las especies extraídas puede ser evaluado tomando como base las entrevistas a la población local en lo que respecta a indicadores seleccionados tales como distribución de clase por tamaño, densidad y frecuencia. De esta manera, puede ser posible conocer si las extracciones, tal como históricamente sucedió, excedían el rendimiento potencial sostenido de las poblaciones. En los bosques de la llanura aluvial inundable del Perú, pocos datos están disponibles para elaborar modelos de crecimiento (por ej. Nebel, 1999), mientras que las evaluaciones de las categorías de las poblaciones de especies que proporcionan PNM han indicado en varias especies una sobre-extracción y un proceso de reducción (Kvist y Nebel, 1999; Kvist et al., 1999a). Con el fin de ayudar a elaborar mejores estimaciones y modelos de rendimiento potencial sostenido de PFNM, en los bosques de la llanura aluvial inundable peruana, es necesario contar con conocimiento adicional, poniendo énfasis en las especies comercialmente valiosas. Además, existe limitado conocimiento sobre la extensión de los hábitats que contienen las especies proveedoras de PFNM, por lo 
que se incide en la necesidad de proporcionar tal información. En muchos casos, la extracción es depredadora, lo que implica que los individuos que proveen el producto son removidos o relegados en la reproducción. Las poblaciones de tales especies son susceptibles de desaparecer, por lo que es obvio la necesidad de desarrollar y promover técnicas de aprovechamiento de bajo impacto así como el mejoramiento de la regeneración y los rodales.

La organización y la reglamentación del acceso y el uso de los recursos base de los PFNM es vital para el uso sostenible de la tierra, poniendo especial atención, básicamente, al comportamiento de los campesinos y otros extractores del recurso. Algunos ejemplos de factores reguladores son las condiciones del mercado, la accesibilidad a los recursos naturales, las habilidades y capacidad técnica de los extractores, los valores culturales, así como los sistemas institucionales y de la propiedad (por ej. Almeida, 1996). Muchos de estos factores ameritan ser tratados con especial atención al relacionarlos con el manejo para producción de PFNM de los bosques de la llanura aluvial inundable del Perú. No se promulgan, formalmente, los derechos permanentes de los usuarios de la tierra. Esto constituye un problema, pues el rol de los sistemas de reglamentación de extracción del recurso, basados en la tradición, está disminuyendo junto con los cambios de los valores culturales de los habitantes de la llanura aluvial inundable, incrementándose actividades que corresponden a intereses externos de motivación comercial. El incentivo para los campesinos, por actuar solidariamente con otros, está debilitado y, en general, los extractores del recurso tienden a no priorizar los beneficios en el largo plazo. Existen ya algunas normas sobre la extracción de ciertos productos, pero éstas -a menudo- no se cumplen debido a la corrupción, ignorancia y falta de instituciones capaces de controlar las actividades de extracción. Bajo estas condiciones, parece importante llevar a cabo una revisión del sistema de propiedad, lo cual es socio-económicamente honesto, dando como resultado la distribución de la tierra para intereses identificados. También es esencial reforzar un apropiado sistema institucional y organizacional con el deseo y la capacidad de promover el manejo y la conservación, especialmente en aquellas tierras comunes donde se desarrollan los PFNM. Un servicio de extensión en buen funcionamiento es otro de los requerimientos para el soporte del uso sostenible de los PFNM.

\subsection{Domesticación de los recursos del bosque de la llanura aluvial inundable}

Es posible la domesticación de ciertas especies forestales de poblaciones naturales que ya no pueden abastecer el mercado con los productos demandados. Sin embargo, 
la domesticación solamente sucede si es técnica, económica y socio-culturalmente factible. Está, muchas veces, precedida de un periodo de sobre-extracción de las poblaciones naturales, debido a que los incentivos económicos para los extractores hacen que continúen sus actividades, a pesar del riesgo de reducir el recurso natural base, y debido a que las normas sobre actividades de extracción son raramente cumplidas en esta etapa (por ej. Homma, 1996; Wilkie y Godoy, 1996). La domesticación de las especies leñosas perennes tiene lugar en los sistemas agroforestales o mediante el establecimiento de plantaciones, mientras que la crianza de animales domésticos, asociada con bosques naturales, puede tomar varias formas, según la categoría de los animales.

La agroforestería ya es ampliamente practicada en las llanuras aluviales inundables peruanas y podría ser la forma dominante de agricultura. Las prácticas de agricultura migratoria aún prevalecen en los cultivos de subsistencia y casi todos los campesinos poseen chacras. Las excepciones son los cultivos como arroz y frijoles en las playas, cerca al curso de los ríos, que son dejadas al descubierto luego de las inundaciones anuales (Hiraoka, 1985b, 1989, 1992; Padoch y de Jong, 1992; de Jong, 1995, 1999; de Jong et al., 1999; Kvist y Nebel, 1999). Sin embargo, la domesticación de especies leñosas perennes o animales tiene un rol menor en la mayoría de las tierras bajo agroforestería de la llanura aluvial inundable del Perú, debido a que en los cultivos migratorios la regeneración natural domina las purmas. La excesiva infestación de los campos con malezas y pestes, después de algunos años de cultivo, constituye una razón importante para dejar de lado las purmas. Algo de domesticación de especies leñosas perennes tiene lugar en las chacras en donde se producen muchos tipos de productos que van desde fruta hasta madera. Los parámetros relacionados a las condiciones ecológicas, así como socio-económicas, determinan las especies utilizadas (de Jong, 1999). El establecimiento de plantaciones puras de especies leñosas perennes y la domesticación de animales, son todavía relativamente raros en las llanuras aluviales inundables peruanas, aunque en estos últimos años se ha incrementado la plantación del arbusto frutal Myrciaria dubia (H.B.K.) Mc. Vaugh (Myrtaceae). La extracción de las limitadas poblaciones naturales de esta especie ya no puede seguir abasteciendo el mercado con suficiente fruto, que es usado en jugos, refrescos y medicina natural, debido principalmente a su alto contenido de ácido ascórbico.

Desde el punto de vista ecológico y técnico, en las llanuras aluviales inundables peruanas sería factible establecer plantaciones con fines madereros y otros productos. La alta fertilidad de los suelos reduce el riesgo de la pérdida de nutrientes del suelo. Una gran limitante para una producción que demanda altas inversiones y que requiere, además, de un largo plazo, es la exposición de muchos lugares de la llanura 
aluvial inundable a la erosión causada por la corriente del río, lo que agudiza la necesidad de considerar seriamente su localización. El mal drenaje y la excesiva duración de la inundación restringe también las áreas aptas para plantaciones y agroforestería. Muchos cultivos se ven afectados negativamente debido al estancamiento del agua o la inundación y solamente una limitada porción de las llanuras aluviales inundables, situadas en las partes altas, es apta para fines agrícolas y agroforestales. La tolerancia al estancamiento de agua o a la inundación de varias especies leñosas perennes frutícolas de las llanuras aluviales inundables, sugiere que se cuente con abundante área disponible para la domesticación de estas especies. Sin embargo, al presente, existe poca información sobre la superficie de las tierras de la llanura aluvial inundable expuesta a diferentes periodos de inundación. Es fundamental, entonces, en el planeamiento del uso de la tierra, proveer tal información y es un prerrequisito para evitar consecuencias ecológicas inadvertidas debido a la conversión de ciertos hábitats en plantaciones homogéneas o rodales agroforestales. Dentro de un contexto socio-económico, es importante estar consciente de que los cultivos en las partes altas de las llanuras aluviales inundables es de gran importancia en las actividades de subsistencia. Por lo tanto, la promoción del uso competitivo de la tierra podría reducir la igualdad socio-económica. De cualquier manera, es necesario y posible mejorar la eficiencia de la producción agrícola actual mediante la introducción de sistemas mejorados de manejo. Una posibilidad puede ser la aplicación de sistemas agroforestales mejorados en purmas que incluye árboles maderables y frutales. Un resultado satisfactorio demandará, probablemente, aplicar una propuesta basada en la participación de la comunidad así como en la eliminación de las dificultades ocasionadas por la incertidumbre en el derecho a la tenencia de la tierra.

\section{PLANEAMIENTO E IMPLEMENTACIÓN DEL USO SOSTENIBLE DE LA TIERRA EN LOS BOSQUES DE LA LLANURA ALUVIAL INUNDABLE}

El presente nivel de vida en la Amazonía Peruana necesita ser mejorado y, en relación a este tema, existe la posibilidad de considerar diversas formas de incrementar el valor de la producción. Las actuales actividades socio-económicas de los habitantes de la llanura aluvial inundable amazónica están concentradas en la agricultura migratoria, la pesca y la extracción de PFNM (por ej. Hiraoka, 1985a, 1985b, 1989, 1992; Bergman, 1990; Padoch y de Jong, 1990; Kvist et al., 1999a, 1999b; de Jong, 1995, 1999; de Jong et al., 1999). El valor estimado de las economías de los campesinos es de 2217 US\$/año (Kvist et al., 1999a). Las actividades basadas en la 
comercialización, que en gran parte son desarrolladas por intereses económicos urbanos, tienden a concentrarse en la extracción maderera y la pesca (Kvist y Nebel, 1999).

Sin embargo, como se indica más adelante, es probable que el valor potencial económico de la producción en las llanuras aluviales inundables sea mucho más elevado. Las mejoras deben ser logradas a través de mejoras de la producción primaria así como de la producción de productos con mayor valor agregado. Un ejemplo es la producción de madera de los bosques naturales de la llanura aluvial inundable. La producción anual actual de madera redonda, en los más de seis millones de hectáreas de las llanuras aluviales inundables de la Amazonía Peruana, es de alrededor de $400000 \mathrm{~m}^{3}{ }^{1}$. Sin aplicar tratamientos silviculturales que mejoren el crecimiento, el incremento anual de los volúmenes en pie de las restingas forestales fue de $2-5 \mathrm{~m}^{3} /$ ha/año, teniendo en cuenta el tipo de bosque y la intensidad de aprovechamiento (Figura 1). Asumiendo que los bosques localizados en solamente el 10\% de las llanuras aluviales inundables puedan sostener una producción de $3 \mathrm{~m}^{3} / \mathrm{ha}$ /año, esto resultaría en un potencial de producción de 1,8 millones $\mathrm{m}^{3} /$ año, lo que indica que existe un considerable potencial de producción no utilizado. Con los beneficios de la extracción de madera, basado en el principio de rendimiento sostenido, sería posible, en 75 ha de bosques de restinga ${ }^{2}$ aproximadamente, obtener un beneficio equivalente a los 2217 US\$/año. Similar potencial existe al incrementar el valor de otras mercancías tanto de subsistencia como de fines comerciales, tal como se indicaba también en las secciones anteriores de este documento.

Tal como sucede en otras partes, el efecto satisfactorio del planeamiento y la implementación del uso sostenible de la tierra en las llanuras aluviales inundables del Perú, depende de un genuino compromiso político que redunde en decisiones firmes y claras, tomadas en un proceso participativo que involucre importantes entes económicos. Si el uso sostenible de la tierra debe ser adecuadamente planificado e

1 Datos de la extracción de madera redonda de especies forestales de la llanura aluvial inundable en los departamentos de Loreto y Ucayali, proporcionados por Kvist y Nebel (1999), sumados; asumiendo que la producción de madera redonda en el departamento de Ucayali se podría estimar expandiendo la producción de madera aserrada, plywood y parquet con un factor que iguale al factor que existe en el departamento de Loreto entre estas mercancías.

2 De la Figura 1 se escogió un escenario con MHD del 80\%, que resulta en beneficios netos del aprovechamiento de la madera de aproximadamente 500 US\$/ha y ciclos de rotación para el rendimiento sostenido de madera de alrededor de 17 años. 
implementado en las llanuras aluviales inundables peruanas, esto demandará una estrategia integral de uso de la tierra que pueda guiar el proceso. En la elaboración de tal estrategia se propone considerar los siguientes tópicos:

- Intervenciones del manejo extensivo pueden ser llevadas a cabo, puesto que los ecosistemas, por naturaleza, están adaptados a grandes perturbaciones.

- $\quad$ Las interferencias, dentro de cada hábitat, deben de ser dispersas y restringidas a una limitada proporción al mismo tiempo, tal como sugería la forma como ocurrían los procesos naturales de la dinámica de la llanura aluvial inundable, lo cual implica también que se debe evitar la conversión en gran escala de ciertos hábitats en otros usos de la tierra.

- Es posible quitar grandes cantidades de nutrientes con la extracción de productos, ya que los suelos son fértiles y periódicamente reciben nuevas remesas de material fértil debido a la sedimentación.

- Se debe restringir o prohibir los sistemas de uso de la tierra que involucren la utilización de pesticidas que dañen el ambiente, así como otros compuestos químicos fácilmente lixiviables y esparcibles hacia las corrientes abiertas de agua.

- Se debe evitar el establecimiento de usos intensivos de la tierra en áreas con alto riesgo de erosión debido a la corriente de los ríos.

- Se debe desarrollar herramientas de manejo y sistemas de uso de la tierra capaces de controlar el desarrollo de recursos uniformes en áreas de pequeña escala.

- Las llanuras aluviales inundables deben ser clasificadas de acuerdo a factores ambientales que determinan áreas de producción y de conservación (por ej. periodos de inundación, situación del drenaje, tipo de hábitat, riesgo de erosión).

- Se debe definir e implementar un sistema de tenencia de la tierra que comprenda las llanuras aluviales inundables, sin considerar el carácter geográficamente dinámico del medio ambiente.

- Se debe reforzar e incrementar las investigaciones concernientes a las posibilidades y problemas más relevantes de los diferentes usos de la tierra.

- Se deben crear y apoyar instituciones y organizaciones capaces de colaborar con el manejo de las llanuras aluviales inundables. 


\section{RECONOCIMIENTOS}

Me gustaría agradecer a todas las personas que cordialmente apoyaron o participaron en la conducción del trabajo de campo a fin de crear la base para redactar este artículo. El Instituto de Investigaciones de la Amazonía Peruana (IIAP) gustosamente proporcionó su apoyo y sus instalaciones en Perú. Estoy en deuda con el personal del IIAP y con muchas otras personas por sus comentarios fructíferos. Lars Peter Kvist, Jens Dragsted y especialmente Anders Ræbild comentaron las primeras versiones de este manuscrito. El financiamiento fue proporcionado por la Agencia Danesa de Desarrollo Internacional (DANIDA).

\section{BIBLIOGRAFIA}

ALMEIDA, M.W.B. 1996. Household extractive economies. In: J.E.M. Arnold; M.P. Ruiz (editors). Current issues in nom-timber forest products research. CIFOR/ODA, Bogor. pp. 119-142.

ARMSTRONG, W.; Brandle, R.; Jackson, M.B. 1994. Mechanisms of floor tolerance in plants. In: Acta Botanica Neerlandica, 43(4): 307-358.

BARROS, A.C.; UHL, C. 1995. Logging along the Amazon river and estuary: patterns, problems and potential. In: Forest Ecology and Management, 77:87-105.

BERGMAN, R. 1990. Economía amazónica. Estrategias de subsistencia en las riberas del Ucayali en el Perú. Lima: Centro Amazónico de Antropología y Aplicación Práctica. 209 pp.

COLLIER, R. 1981. Jague al Batón. La historia del caucho en la Amazonía. Lima: Centro Amazónico de Antropología y Aplicación Práctica. 259 pp.

CRAWFORD, R.M.M. 1982. Physiological responses to flooding. In: Enc. Plant. Physiol., 12B: 453-477.

JONG, W. de. 1995. Diversity, variation, and change in ribereño agriculture and agroforestry. Den Haag: CIP-DATA Koninklijke Biliotheek. 168 pp. 
1999. Tree and forest management in the floodplains of the peruvian Amazon. Bogor: Center for International Forestry Research. Unpublished manuscript.

JONG, W. de; FREITAS, L.; BALUARTE, J.; KOP, P. van de; SALAZAR, A.; INGA, H.; MELÉNDEZ, W.; GERMANÁ, C. 1999. Secondary forest dynamics in the Amazon floodplain in Peru. Bogor: Center for International Forestry Research. Unpublished manuscript.

DENEVAN, W.M. 1976. The aboriginal population of Amazonia. In: W.M. Denevan (editor). The native population of the Americas in 1492. Wisconsin: The University of Wisconsin Press. pp. 205-234.

DOUROJEANNI, W.M. 1990. Amazonía ¿que hacer? Iquitos: Centro de Estudios Teológicos de la Amazonía. 444 pp.

FAGERIA, N.K.; WRIGHT, R.J.; BALIGAR, V.C.; SOUZA, C.M.R. 1991. Characterization of physical and chemical properties of varzea soils of Goias state of Brazil. In: Commun. Soils Sci. Plant. Anal., 22(15\&16): 1631-1646.

FAO. 1993. Management and conservation of closed forest in tropical America. In: FAO Forestry Papers, $\mathrm{N}^{\circ} 101$. Rome. $141 \mathrm{pp}$.

FOSTER, R.B. 1990. The floristic composition of the río Manu floodplain forest. In: A.H. Gentry (editor). Four neotropical rainforests. New Haven: Yale University Press. pp. 99-111.

FOSTER, R.B.; ARCE, J.B.; WACHTER, T.S. 1986. Dispersal and the sequential plant communities in Amazonian Peru floodplain. In: A. Estrada; T.H. Fleming (editors). Frugivores and seed dispersal. Dordrecht: Dr. W. Junk Publishers. pp. 357-370.

FREITAS, L.A. 1996. Caracterización florística y estructural de cuatro comunidades boscosas de la llanura aluvial inundable en la zona Jenaro Herrera, Amazonía Peruana. Documento Técnico Nº 21. Iquitos: Instituto de Investigaciones de la Amazonía Peruana. 73 pp.

FURCH, K. 1997. Chemistry of várzea and igapó soils and nutrient inventory of their floodplain forests. In: W.J. Junk (editor). The central Amazon floodplain. Ecology of pulsing system. Berlin: Springer-Verlag. pp. 47-68. 
GENTRY, A.H.; TERBORGH, J. 1990. Composition and dynamics of the Cocha Cashu "mature" floodplain forest. In: A.H. Gentry (editor). Four neotropical rainforests. New Haven and London: Yale University Press. pp. 542-563.

GILL, C.J. 1970. The flooding tolerance of woody species: A review. In: Forestry Abstracts, 3(4): 671-688.

GOTTSBERGER, G. 1978. Seed dispersal by fish in the inundated region of Huamaita, Amazonia. In: Biotropica, 10(3): 170-183.

GOULDING, M. 1980. The fishes and the forest. Explorations in Amazonian history. Berkeley: University of California Press. 289 pp.

GOULDING, M. 1983. The role of fishes in seed dispersal and plant distribution in Amazonian floodplain ecosystems. In: Sonderd naturwiss Ver Hamburg, 7: 271-283.

1985. Forest fishes of the Amazon. In: G.T. Prance; T.E. Lovejoy (editors). Amazonia. Oxford: Pergamon Press. pp. 267-276.

GOULDING, M.; SMITH, N.J.H.; MAHAR, D.J. 1996. Floods of fortune. Ecology and economy along the Amazon. New York: Columbia University Press. $193 \mathrm{pp}$.

HIRAOKA, M. 1985a. Mestizo subsistence in riparian Amazonia. In: National Geographic Research, 1(2): 236-246.

1985b. Cash cropping, wage labor, and urban ward migrations: changing floodplain subsistence in the peruvian Amazon. In: Studies in Third World Countries, 32: 199-243.

1989. Ribereños changing economic patterns in the peruvian Amazon. Journal of cultural Geography, 9(2): 103-119.

1992. Caboclo and ribereño resource management in Amazonia: A review. In: K.H. Redford; C. Padoch (editors). Conservation of Neotropical forest. Working from traditional resource use. New York: Columbia University Press. pp. 134-157. 
HOMMA, A.K.O. 1996. Modernization and technological dualism in the extractive economy of Amazonia. In: J.E.M. Arnold; M.P. Ruiz (editors). Current issues in non-timber forest products research. CIFOR/ODA, Bogor, pp. 59-83.

HOOK, D.D. 1984. Adaptations to flooding with fresh water. In: T.T. Kozlowski (editor). Flooding and plant growth. Academic Press. pp. 265-294.

IRION, G. 1989. Quaternary geologial history of the Amazon lowlands. In: L.B. Holm-Nielsen; I.C. Nielsen; H. Balslev (editors). Tropical forests. Botanical dynamics, speciation and diversity. Academic Press Limited. pp. 23-34.

IRION, G.; JUNK, W.J.; MELLO, J.A.S.N. de. 1997. The large central Amazonian river floodplains near Manaus: geological, climatological, hydrological and geomorphological aspects. In: W.J. Junk (editor). The central Amazon floodplain. Ecology of a pulsing system. Berlin: Springer-Verlag. pp. 23-46.

JUNK, W.J. 1989. Flood tolerance and tree distribution in central Amazonian floodplains. In: L.B. Holm-Nielsen; I.C. Nielsen; H. Balslev (editors). Tropical forest. Botanical dynamics, speciation and diversity. Academic Press Limited. pp. 47-64.

1997. General aspects of floodplain ecology with special reference to Amazonian floodplains. In: W.J. Junk (editor). The central Amazon floodplain. Ecology of a pulsing system. Berlin: Springer-Verlag. pp. 3-20.

JUNK, W.J.; BAYLEY, P.B.; SPARKS, R.E. 1989. The flood pulse concept in riverfloodplain systems. In: D.P. Dodge (editor). Proceedings of the international large river symposium. Can, Spec. Publ. Fish. Aquat. Sci. pp. 110-127.

JUNK, W.J.; PIEDADE, M.T.F. 1997. Plant life in the floodplain with special reference to herbaceous plants. In: W.J. Junk (editor). The central Amazon floodplain. Ecology of a pulsing system. Berli: Springer-Verlag. pp. 147-185.

JUNK, W.J.; SOARES, M.G.M.; SAINT-PAUL, U. 1997. The fish. In: W.J. Junk (editor). The central Amazon floodplain. Ecology of a pulsing system. Berlin: Springer-Verlag. pp. 385-408. 
KALLIOLA, R.; PUHAKKA, M. 1993. Geografía de la selva peruana. In: R. Kalliola; M. Puhakka; W. Danjoy (editors). Amazonía peruana. Vegetación húmeda tropical en el llano subandino. Jyvaskyla: PAUT/ONERN. pp. 9-22.

KUBITZKI, K. 1989. The ecogeographical differentiation of Amazonian inundation forest. In: Pl. Syst. Evol., 162: 285-304.

KVIST, L.P.; ANDERSEN, M.K.; HESSELSOE, M.; VANCLAY, J. 1995. Estimating use-values and relative importance of Amazonian flood plain trees and forests to local inhabitants. In: Commonwealth Forestry Review, 74(4): 293-300.

KVIST, L.P.; NEBEL, G. 1999. A review of peruvian flood plain forests: Ecosystems, inhabitants and resource use. Copenhagen: Royal Veterinary and Agricultural University, Department of Economics and Natural Resources. Unit of Forestry. Unpublished manuscript.

KVIST, L.P.; ANDERSEN, M.K.; STAGEGAARD, J.; HESSELSOE, M.; LLAPAPASCA, C. 1999a. Extraction from woody forest plants in flood plain communities in Amazonian Perú: Evaluation, choice and conservation status of resources. Copenhagen: Royal Veterinary and Agricultural University, Department of Economics and Natural Resources. Unit of Forestry. Unpublished manuscript.

KVIST, L.P.; GRAM, S.; CÁCERES, A.C.; ORÉ, J.B. 1999b. Socio-economy of villagers in the peruvian Amazon with a particular focus at extraction. A comparison of seven flood plain communities along the lower Ucayali and Marañon rivers. Copenhagen: Royal Veterinary and Agricultural University, Department of Economics and Natural Resources. Unit of Forestry. Unpublished manuscript.

MEGGERS, B.J. 1971. Amazonía. Man and culture in a counterfeit paradise. Illinois: AHM Publishing Corporation. 182 pp.

MORAN, E.F. 1989. Models of native and folk adaptation in the Amazon. In: Advances in Economic Botany, 7: 22-29.

MUÑIZ-MIRET, N.; VAMOS, R.; HIRAOKA, M.; MONTAGNINI, F.; MENDELSOHN, R. O. 1996. The economic value of managing the acai 
palm (Euterpe oleracea Mart.) in the floodplains of the Amazon estuary, Pará, Brazil. In: Forest Ecology and Management, 87: 163-173.

NEBEL, G. 1999. Minquartia guianensis Aubl.: Use, ecology and management in forestry and agroforestry. Copenhagen: Royal Veterinary and Agricultural University, Departament of Economics and Natural Resources. Unit of Forestry. Unpublished manuscript.

NEBEl, G.; KVIST, L.P.; VANCLAY, J.K.; VIDAURRE, H. 1999a. Forest dynamics in flood plain forest in the peruvian Amazon: Effects of disturbance and implications for management and conservation. Copenhagen: Royal Veterinary and Agricultural University, Department of Economics and Natural Resources. Unit of Forestry. Unpublished manuscript.

NEBEL, G.; DRAGSTED, J.; SALAZAR, A.V. 1999b. Litter fall, biomass and net primary production in flood plain forests in the peruvian Amazon. Copenhagen: Royal Veterinary and Agricultural University, Department of Economics and Natural Resources. Unit of Forestry. Unpublished manuscript.

NEBEL, G.; DRAGSTED, J.; SIMONSEN, T.R.; VANCLAY, J.K. 1999c. The Amazon flood plain forest tree Maquira coriacea (Karsten) CC. Berg: Aspects of ecology and management. Copenhagen: Royal Veterinary and Agricultural University, Department of Economics and Natural Resources. Unit of Forestry. Unpublished manuscript.

PADOCH, C.; JONG, W. de. 1990. Santa Rosa: The impact of the forest products trade on an Amazonian place and population. In: Advances in Economic Botany, 8: 151-158.

1992. Diversity, variation, and change in ribereño agriculture. In: K.H. Redford; C. Padoch (editors). Conservation of neotropical forest. Working from traditional resource use. New York: Columbia University Press. pp. 158-174.

PENNANO, G. 1988. La economía del caucho. Iquitos: CETA. 264 pp.

PETERMANN, P. 1997. The birds. In: W.J. Junk (editor). The central Amazon floodplain. Ecology of a pulsing system. Berlin: Springer-Verlag. pp. 419-452. 
PETERS, C.M.; GENTRY, A.H.; MENDELSOHN, R.O. 1989. Valuation of an Amazonian Rainforest. In: Nature, 339: 655-656.

RÄSÄNEN, M. 1993. La geohistoria de vegetación en la selva baja peruana. In: R. Kalliola; M. Puhakka; W. Danjoy (editors). Amazonía peruana. Vegetación húmeda tropical en el llano subandino. Jyvaskyla: PAUT/ONERN. pp. 43-68.

RÄSÄNEN, M.E.; NELLER, R.; SALO, J.; JUNGNER, H. 1992. Recent and ancient fluvial deposition systems in the Amazonian foreland basin, Peru. In: Geol. Mag, 129(3): 293-306.

RÄSÄNEN, M.E.; SALO, J.; KALLIOLA, R. 1987. Fluvial perturbance in the Western Amazon basin: regulation by long-term sub-Andean tectonics. In: Science, 238: 1398-1401.

ROOSEVELT, A. 1989. Resource management in Amazonia before the conquest: Beyond ethnographic projection. In: Advances in Economic Botany, 7: 30-62.

SALO, J.; KALLIOLA, R.; HAKKINEN, I.; MAKINEN, Y.; NIEMELA, P.; PUHAKKA, M.; COLEY, P.D. 1986. River dinamics and the diversity of Amazon lowland forest. In: Nature, 322: 254-258.

SALO, J.; RASÄNEN, M.E. 1989. Hierarchy of landscape patterns in western Amazon. In: L.B. Holm-Nielsen; I.C. Nielsen; H. Balslev (editors). Tropical forest Botanical dynamics, speciation and diversity. Academic Press. pp. 239-251.

SCHLÜTER, U.B.; FURCH, V.B.; JOLY, C.A. 1993. Physiological and anatomical adaptations by young Astrocaryum jauari Mart. (Arecaceae) in periodically inundated biotopes of Central Amazonia. In: Biotropica, 25(4): 384-396.

SOINI, P.; SICCHAR, L.A.; GIL, N.G.; FACHIN, T.A.; PEZO, R.; CHUMBE, A.M. 1996. Una evaluación de la fauna silvestre y su aprovechamiento en la Reserva Nacional Pacaya-Samiria, Perú. Documento Técnico N² 24. Iquitos: Instituto de Investigaciones de la Amazonía Peruana. 64 pp. 
VERISSIMO, A.; BARRETO, P.; MATTOS, M.; TARIFA, R.; UHL, C. 1992. Logging impacts and prospects for sustainable forest management in an old Amazonian frontier: the case Paragominas. In: Forest Ecology and Management, 55: 169-199.

WILKIE, D.S.; GODOY, R.A. 1996. Trade, indigenous rain forest economies and biological diversity - model predictions an directions for research. In: J.E.M. Arnold; M.P. Ruiz (editors). Current issues in non-timber forest products research. Bogor: CIFOR/ODA. pp. 83-102.

WORBES, M. 1997. The forest ecosystem of the floodplains. In: W.J. Junk (editor). The central Amazon floodplain. Ecology of a pulsing system. Berlin: Springer-Verlag. pp. 223-266.

WORBES, M.; KLINGE, H.; REVILLA, J.D.; MARTIUS, C. 1992. On the dynamics, floristic subdivision and geographical distribution of várzea forests in Central Amazonia. In: Journal of Vegetation Science, 3: 353-564. 

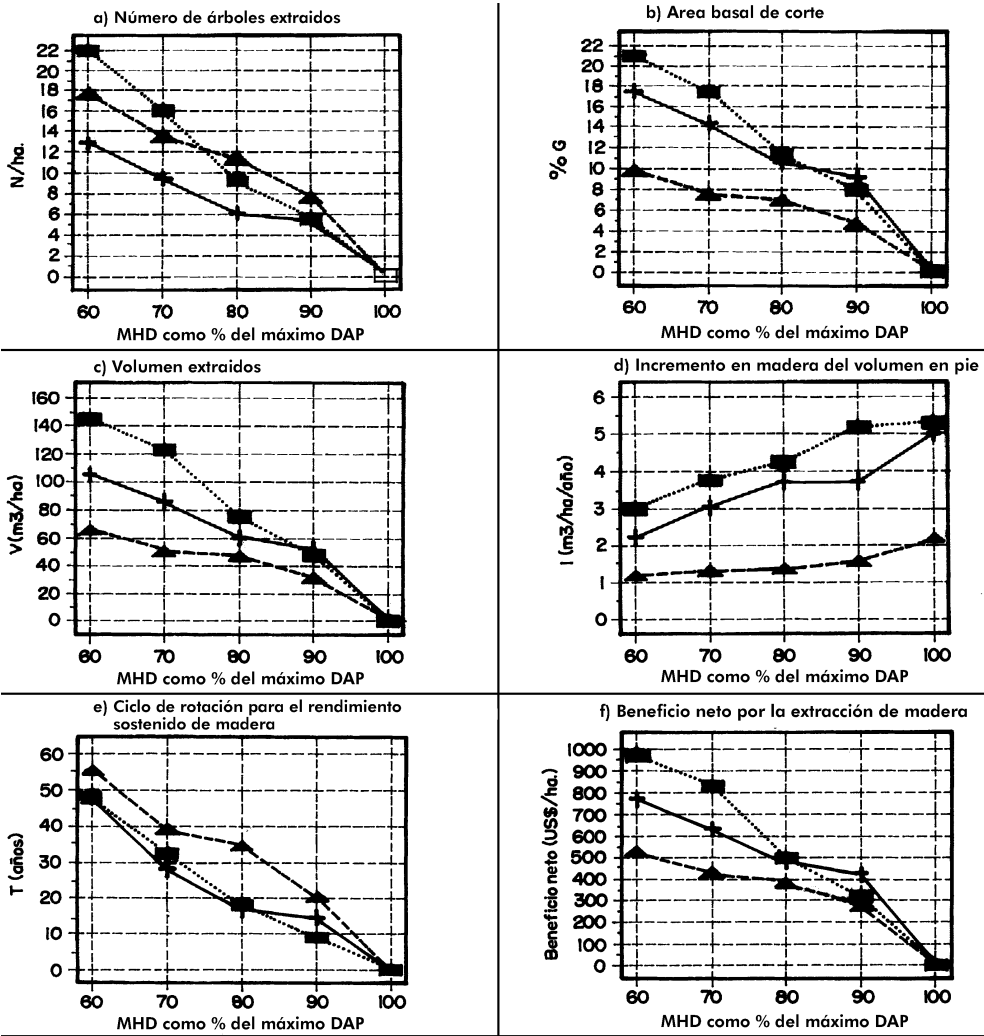

f) Beneficio neto por la extracción de madera
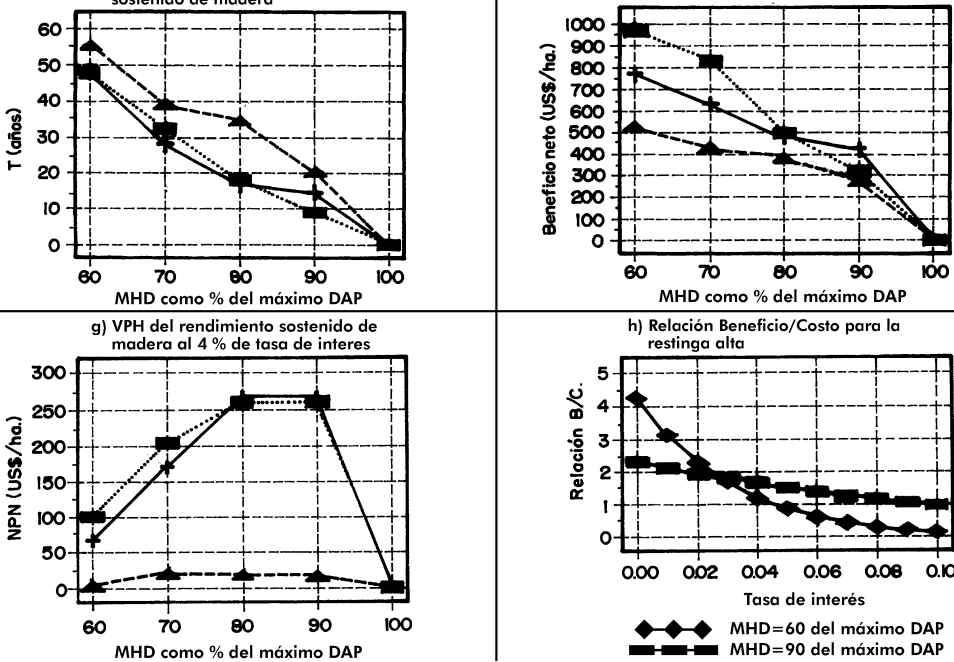

h) Relación Beneficio/Costo para la restinga alta

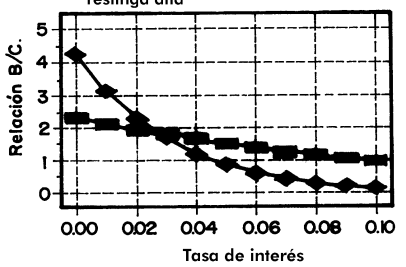

HD como \% del máximo DAP

Fig. 1. Importantes parámetros para escenarios que muestran diferentes intensidades de aprovechamiento en concordancia con el diámetro mínimo de corte (DMC) como porcentaje del máximo diámetro a la altura del pecho (DAP) de especies comerciales en tres bosques peruanos de llano inundable (cuadrado = restinga alta; triángulos = restinga baja; cruces $=$ tahuampa). 